\title{
Civilisations
}

Revue internationale d'anthropologie et de sciences

humaines

49 | 2002

Pain, fours et foyers des temps passés

\section{L'offrande céréalière dans les rituels funéraires du sud-est européen}

Marianne Mesnil et Assia Popova

\section{OpenEdition \\ Journals}

Édition électronique

URL : http://journals.openedition.org/civilisations/1412

DOI : 10.4000/civilisations. 1412

ISSN : 2032-0442

\section{Éditeur}

Institut de sociologie de l'Université Libre de Bruxelles

\section{Édition imprimée}

Date de publication : 3 juin 2002

Pagination : 101-117

ISBN : 0009-8140

ISSN : 0009-8140

Référence électronique

Marianne Mesnil et Assia Popova, «L'offrande céréalière dans les rituels funéraires du sud-est européen », Civilisations [En ligne], 49 | 2002, mis en ligne le 01 juin 2005, consulté le 19 avril 2019. URL : http://journals.openedition.org/civilisations/1412; DOI : 10.4000/civilisations.1412

Ce document a été généré automatiquement le 19 avril 2019

(c) Tous droits réservés 


\title{
L'offrande céréalière dans les rituels funéraires du sud-est européen
}

\author{
Marianne Mesnil et Assia Popova
}

\section{NOTE DE L'AUTEUR}

La notation scientifiquement correcte des vocables des langues qui utilisent des alphabets non-latin exige une translitération normalisée. Dans nos textes, les termes bulgares et russes sont translitérés du cyrillique et les termes roumains sont presentés comme on les écrit. Dans la translitération normative la lettre c correspond au phonème/ts/, écrit par les roumains / $\mathrm{T}+$ signe/. Ainsi le mot colac pris pour translitéré se lit « tsolats » et le mot coliva donne «tsoliva ». C'est pour cela que je milite corps et âme pour la notation cyrillique. En tout état de cause, la lettre « $\mathrm{c}$ » en raison de sa duplicité est à éviter, dans la mesure du possible. Pour les mots d'origine grecque, on prend la translitération du grec ou la lettre $\mathrm{k}$ correspond à un seule phonème $/ \mathrm{k} /$. Le cas $\mathrm{C} / \mathrm{k} / \mathrm{T}$ n'est pas le seul inconvénient de translitération ...

\section{De l'ethnologie à l'archéologie}

1 Parmi les traits distinctifs qui permettent tant soit peu de tracer une frontière entre " animalité » et « humanité », on peut sans conteste faire figurer les pratiques liées à la mort : pleurer et enterrer ses morts est bien le propre de l'homme. Il n'est pas une société humaine qui n'ait entouré cet évènement de tout un appareil de prescriptions et d'interdits réglant de manière souvent minutieuse ce dangereux moment durant lequel la société des vivants se trouve confrontée à un corps qui lui devient soudainement "étranger », un cadavre. Toute société s'applique à transformer l'événement biologique de la mort en une séquence culturelle complexe : ce sont les rites de deuil. C'est pourquoi on peut considérer que de tous temps, les objets ou fragments d'objets conservés dans les tombes ont pris place dans une séquence rituelle. L'approche ethnologique consiste à 
étudier de tels objets par rapport à ces séquences qui leur donnent sens. Cette démarche peut-elle, par analogie, suggérer un contexte qui échappe nécessairement aux archéologues confrontés aux objets ou traces d'objets sur lesquels ils ont à travailler?

\section{Un culte des morts dans les Balkans}

Pour nous conformer au thème de cette rencontre, notre propos se limitera à un seul aspect des rites mortuaires pratiqués encore de nos jours dans l'aire culturelle de l'orthodoxie balkanique: les offrandes céréalières. On n'aura cependant à l'esprit que celles-ci ne peuvent être dissociées dans la pratique, de deux autres offrandes "fondamentales", celle de lumière (généralement sous forme de cierges) et celle des libations (d'eau et de vin) pour "la soif du mort». Par ailleurs, il faut insister sur le foisonnement de pratiques et croyances touchant à la mort dans cette partie de l'Europe (et tout particulièrement en Roumanie) dont nous ne pourrons qu'entrevoir ici la complexité. Nous y distinguerons donc deux grandes catégories d'offrandes céréalières, selon qu'elles s'insèrent dans la séquence rituelle accomplie durant la période qui suit le décès d'un individu, ou qu'elles prennent place dans le cycle de commémorations des morts que l'on évoque collectivement à des dates précises d'un calendrier annuel. Mais avant d'aborder ces deux types de cérémonies, il nous faut préciser en quoi les nourritures céréalières qui y interviennent peuvent être qualifiées d'offrandes.

\section{L'institution de la « pomana »}

Il s'agit en effet de rendre compte d'une pratique essentielle des rites de morts dans l'aire balkanique : nous la désignerons ici du terme de pomana, dans le sens que lui donnent les traditions roumaines qui, comme nous allons le voir, en ont érigé la pratique en véritable institution « d'offrandes aux ancêtres ». Le terme pomana est dérivé du slavon, traduction littérale du terme grec mnemè (mémoire, souvenir) et qui désigne précisément les fêtes de commémorations des morts. Mais en roumain, le mot s'applique à la fois à la cérémonie destinée à honorer un ou des morts, et aux objets distribués à cette occasion en leur mémoire.

En quoi, dès lors, ces dons peuvent-ils être qualifiés d'offrandes?

$4 \mathrm{Au}$ sens strict, le terme d'offrande implique un destinataire transcendantal. Peut-on dès lors parler d'une " offrande aux morts " ? Oui, dans la même mesure où nous ne pensons pas excessif de parler d'un "culte des ancêtres » dans l'aire culturelle balkanique. Ce culte s'intègre bien sûr dans un système de croyances et pratiques chrétiennes ou, selon l'opposition de S. Reinach, on ne prie plus les morts, mais on prie « pour les mort $»^{1}$. Il n'en reste pas moins qu'il s'agit d'un véritable culte qui repose sur des croyances et une représentation de l'espace de l'au-delà qui n'a pas grand chose à voir avec les représentations chrétiennes du paradis et de l'enfer; ce culte possède également un calendrier de commémorations dont les moments forts se confondent avec les grandes célébrations de la liturgie chrétienne : ce sont les « fêtes d'ancêtres" (en roumain Mosi) ou « fêtes de commémoration des âmes » (en bulgare, zadusnitsî) ${ }^{2}$ au cours desquelles ont lieux ces rites d'offrandes (voir ci-après). Par ailleurs ces « dons » sont nécessairement adressés aux morts (éventuellement à des saints, mais surtout aux morts d'un lignage), et jamais à Dieu. Et si l'autel sert à consacrer ces dons de nourritures, il n'est cependant 
qu'un lieu de transit, le geste rituel du don ayant lieu, quant à lui, sur les tombes. Ainsi, pour ce " culte des ancêtres ", c'est la tombe elle-même qui fait office d'autel. Mais ce qui fait encore la spécificité de la pomana, et qui a pu la faire confondre avec l'aumône, c'est qu'elle doit être reçue par un tiers (vivant) « au nom du mort ». De fait, le propre de cette offrande est de réaliser une médiation entre un vivant et un mort, par l'action de "donner à un tiers ». A la base de ce système de "transferts ", existe la croyance en une véritable "transubstantiation" de ces objets qui, tout en profitant aux vivants qui les reçoivent ici-bas, bénéficient au mort à titre individuel, dans l'au-delà : l'acte de donner et recevoir entre vivants "au nom du mort », permet le transfert de l'objet offert et en quelque sorte «spiritualisé » jusqu'au pays des ancêtres ${ }^{3}$.

\section{Des céréales bonnes à manger et bonnes à penser}

5 La place des préparations céréalières dans ces rituels d'offrandes funéraires est particulièrement importante. On sait en effet combien le blé, qu'il soit à l'état «naturel » sous forme de grain, ou au contraire, hautement élaboré sous forme de pain richement décoré, permet de penser la vie et la mort. Ainsi cette réflexion recueillie sur le terrain en Bulgarie, à propos de la période où les morts sont censés visiter les vivants:

6 "Les âmes des morts, quand elles sont sur terre, doivent recevoir de la nourriture des vivants. C'est pourquoi les gens distribuent régulièrement de la nourriture « pour les âmes de leurs morts " pendant cette période. On croit que si les vivants ne distribuent pas de la nourriture pour leurs morts, les âmes mangent la fleur du blé et on n'aura pas de bonne récolte $»^{4}$.

7 Cette information indique bien le rapport de solidarité établi entre vivants et morts : des seconds, dépend la fertilité des premiers; et si les ancêtres sont les garants de la force vitale des vivants, ceux-ci ont envers eux le devoir de donner les "pomane ». Au sein d'une culture où la figure du Christ-pain cohabite avec celle d'ancêtres consommateurs d' offrandes, on ne s'étonnera pas de la fréquence avec laquelle nous rencontrons le blé sous toutes ses formes : tant pour les morts que pour les vivants, il apparait comme nourriture privilégiée à même de penser et organiser pour une large part, le rapport entre le monde d'ici et l'au-delà.

\section{Les offrandes céréalières : du grain au pain}

\section{Le Grain}

On trouve l'usage de grains de céréales à plusieurs moments des rites funéraires (voir plus loin); utilisé à l'état «naturel », le grain de blé apparaît comme prototype de la semence (la sperma des Grecs).

\section{Blé bouilli ou kollyva}

Sous l'appelation de kollyva (néogrec), kolivo (Bulgarie) ou coliva (Roumanie), on désigne une préparation de blé concassé, bouilli et généralement sucrés. Dans la série des préparations destinées aux morts, c'est l'aliment «marqué » par excellence, puisque sa consommation est strictement réservée à un usage funéraire. Selon les prescriptions 
liturgiques orthodoxes, «Le blé bouilli dont se compose la kollyva signifie la part morte de la nature humaine - et la part de résurrection des morts ». Cette interprétation cite en référence la parole du Christ selon laquelle «le grain de blé que l'on jette dans la terre, s'il ne pourrit pas, reste seul, et s'il pourrit, amène beaucoup de fruits $»^{6}$.

Dans la mesure où l'on trouve mention d'une telle préparation dans des textes de l'Antiquité, il semble autorisé de considérer cette nourriture rituelle comme un phénomène de "longue durée». Le terme grec de kollyva dont nous adoptons ici l'orthographe translittérée est sans doute une dérivation du vocable kolluba désignant une sorte de gâteau ou bonbon; ce terme aurait lui-même donné par la suite le sens de kollubos : petite pièce de monnaie ${ }^{7}$.

\section{Pains azymes et pains levés}

11 Il faut préciser ici que l'opposition entre " pain azyme et pain levé » telle qu'on la connait dans la tradition chrétienne occidentale, ne peut être transposée dans le contexte de la tradition orthodoxe. On se souviendra du différend qui oppose les deux Eglises à propos du pain de l'Eucharistie (" hostie azyme » chez les uns ; « anaphora » de pain levé chez les autres). Au sein de la culture balkanique qui participe de cette tradition chrétienne orientale, il ne peut être question de considérer la fermentation du pain levé comme synonyme de d'impureté, de putréfaction et de mort ; l'opposition entre les deux types de préparations est ailleurs; elle ne peut prendre sens que référée aux circonstances rituelles de leur utilisation.

\section{Les pains azymes}

12 Le pain azyme est une galette faite d'une pâte céréalière sans levain et vite cuite sous la cendre, ou dans le « four mobile $»^{8}$ ou encore sur le couvercle d'un tel four ; la rapidité de sa fabrication lui a parfois valu l'appellation, en bulgare, de "pain rapide» ou " pain de voyage ", un pain qui ne fait l'objet d'aucune décoration; mais on précise parfois (comme au nord-est de la Bulgarie), qu'il est préparé avec l'eau qui a servi à laver le mort. On le rompt encore brûlant au-dessus de la tête du défunt, et l'on pense que c'est à ce moment que son âme s'envole et reste encore un temps à rôder autour de la maison'. C'est ici l'idée de l'urgence qui domine et dicte l'usage rituel de cette galette; la vapeur qui s'en échappe est censée, par analogie, faciliter la sortie de l'âme du corps. En dehors de ce contexte rituel, il est déconseillé de rompre et de manger du pain très chaud, car on risquerait « de lui faire rendre l'âme ».

\section{Les pains levés}

Les pains levés sont faits d'une pâte à laquelle on incorpore du levain confectionné avec un peu de pâte soustraite à la masse précédente. Contrairement à la fabrication du pain azyme, celle du pain levé demande du temps: long pétrissage puis mise au repos de la pâte, assurant sa "levée » et enfin cuisson dans un four qui assure température et ventilation optimales pour que la pâte ne "retombe" pas. Mais ce processus fait également apparaître l'idée de continuité qu'assure le levain; ce qui explique que cette " chaîne du levain » doive parfois être rompue par prescription rituelle, en particulier au moment du décès d'un membre de la famille ${ }^{10}$. 
Contrairement à la kollyva, les pains levés ne constituent pas une catégorie de nourriture à usage spécifiquement rituel si ce n'est lorsqu'ils sont expressément "marqués » du " sceau christique " $^{11}$ ou bien lorsqu'ils sont décorés de dessins en relief de pâte modelée - Sans être sanctifiés comme le pain de l'eucharistie, ces pains vont cependant « transiter " par l'église pour recevoir la bénédiction du prêtre ; ainsi sacralisés, ils seront offerts aux morts en pomana. Il s'agit donc de pains bénis qui, par analogie avec le pain eucharistique, sont parfois appelés comme lui, prosfora (en grec et en bulgare), prescura (en roumain, emprunté au slavon proskura) ou du diminutif prosforiasan hljab, litt. « petit pain prosphoré » (bulg.).

Fig. 1 : Sceau du pain de l'Eucharistie (prosphora) de l'Eglise orthodoxe bulgare

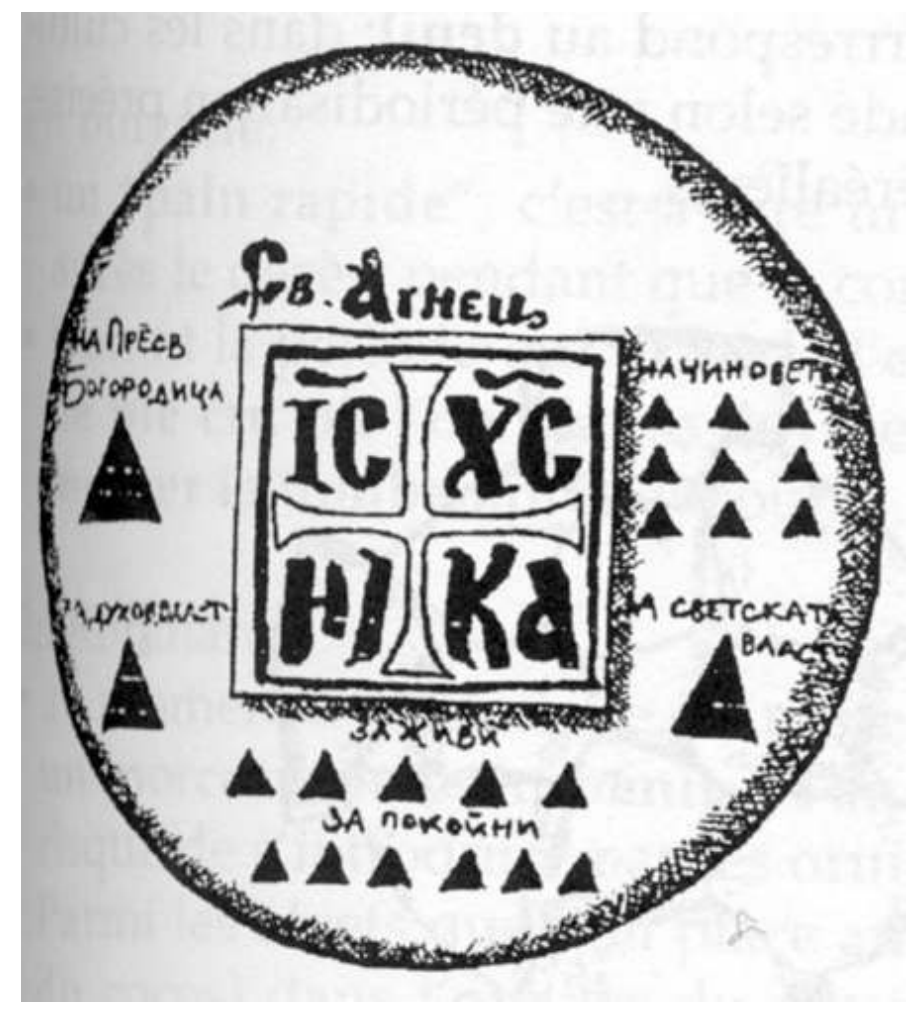

\section{Anneaux, gimblettes et autres croissants}

D'autres pains réservés aux offrandes reçoivent une forme et /ou un nom spécifique qui renvoie à un rite ou une célébration particulière : c'est par exemple le cas des pains des "Quarante martyrs » dont la fête a lieu le 9 mars : ils sont en forme d'oiseaux, d'échelle, de chevaux, de bébés etc. ${ }^{12}$ Tel est le cas aussi des pains bulgares surnommés pains du Saint - Archange. Parfois on fait figurer au centre de tels pains un cercle ouvert confectionné dans un boudin de pâte (Bulgarie du Nord). Nous reviendrons à cette signification des cercles, portions de cercle, ou formes « inachevées » à propos des pains et des kollyva offerts au cours du cycle de deuil.

Par ailleurs, il faut encore tenir compte pour l'interprétation de nos pains rituels, du fait que la même forme voire le nom qui leur est donné peuvent parfois aussi concerner des usages variés et ne constituent donc pas nécessairement une " marque » de leur vocation d'offrande aux morts. Ainsi, le mot kolak (bulg) (colac roum., emprunté au précédent), qui 
nous renvoie à l'idée de cercle, est le terme générique qui désigne les petits pains faits de pâte tressée en anneau ("gimblettes») qui figurent le plus souvent au nombre des offrandes funéraires, mais dont on récompense aussi bien les quêteurs de Noël (chanteurs de calendes).

\section{Faire les morts et les ancêtres}

17 Un objet rituel ne prend sens que par rapport au contexte dont il fait partie. Dans le cas des offrandes funéraires, il s'agit donc de situer dans les grandes lignes ce qu'est une « séquence rituelle » funéraire dans les traditions bulgaro -roumaines dont nos pains sont extraits.

\section{Le deuil du mort et la mémoire des ancêtres : Les rites de la période de deuil}

Si l'on considère l'ensemble des rites funéraires liés à un décès, ceux-ci peuvent être présentés, selon le schéma proposé par A. Van Gennep, en une séquence rituelle en trois temps (séparation, marge et agrégation) qui s'étend sur plusieurs mois, voire plusieurs années. C'est la période qui corrrespond au deuil ; dans les cultures balkaniques, ce temps de «passage » est scandé selon une périodisation précise, par des rites où prennent place des offrandes céréalières.

Fig. 2 : a. «Pain à la croix ", vil. Prevala rég. de Montana, Bulgarie. Pain d'enterrement et de la période de deuil (d'après S. Yaneva, 1989b). b. " Pain à la croix ", vil. Gorni Lom, rég. de Vidin, Bulgarie. Pain d'enterrement et de la période de deuil (d'après S. Yaneva, 1989b)
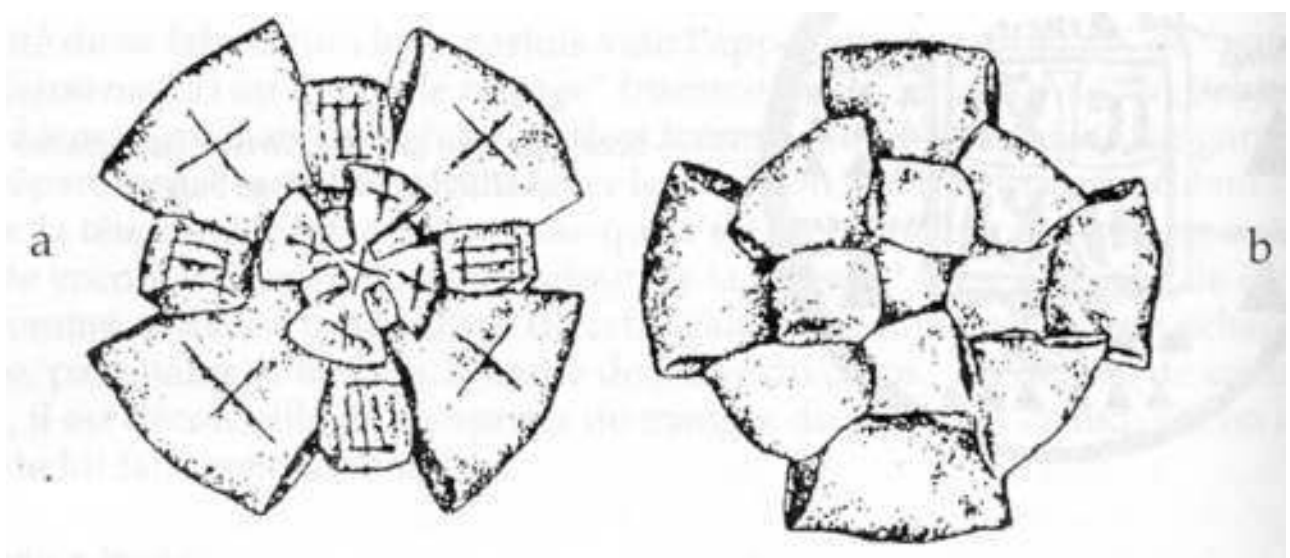

Voici donc tracé dans ses grandes lignes, ce «voyage» du monde d'ici à l'au-delà qu'entreprend le défunt ; c'est la succession des actes rituels effectués par la communauté des vivants, et plus particulièrement son lignage, qui va lui permettre de se transformer successivement de cadavre en mort et de mort en ancêtre. 


\section{Le moment du décès et les préparatifs d'enterrement}

\section{Les trois premiers jours du décès}

Le corps du défunt reste exposé à la maison durant 3 jours. Ils sont dominés par les mesures de « séparation ». Ce sont des mesures qui visent à protéger le corps que l'âme a quitté (cf. veillée du corps - protection des ouvertures du corps) et à donner à l'âme ce dont elle a besoin, en particulier, eau et lumière, tout en lui permettant de "circuler " (ouvrir les fenêtres, ne pas balayer ...) Par ailleurs, il s'agit de protéger les vivants de la contagion de la mort.

Fig. 3 : « Pain du dernier bain », vil. Vinarovo, Bulgarie (d'après Staneva)

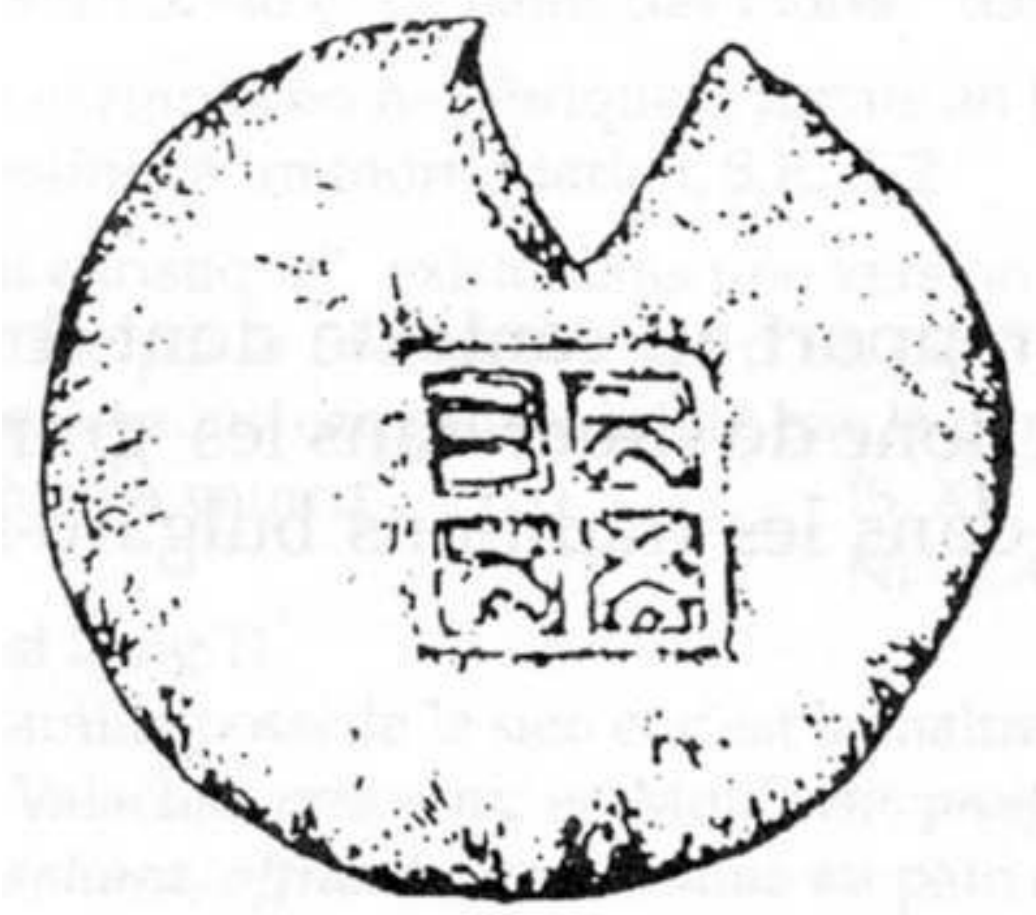

\section{Préparations céréalières}

En Bulgarie :

- Un " pain rapide ", c'est-à-dire un pain azyme, est confectionné immédiatement après le décès, pendant que le corps est encore chaud (voir plus haut) ${ }^{13}$

- Durant la période qui précède l'enterrement, on place à la tête du mort, un bol de blé cru où l'on plante des cierges ; on prélèvera de ces grains de quoi ensemencer la tombe après 40 jours.

En Roumanie :

- Au moment de la toilette du mort, on a soin de mettre dans la bouche du défunt, un morceau de pain bénit à Pâques, qui sert de protection contre le diable qui risque de s'introduire par les orifices du corps pour s'emparer de l'âme.

- Parmi les objets que l'on place au nombre de 9 (correspondant aux 9 ouvertures du corps) dans l'oreiller du mort qui garnira le cercueil, figurent 9 grains de millet. Le millet (comme 
les noix qui figurent aussi dans cette liste d'objets) serait ici symbole de fécondité (Bernabé :39).

- Partout, on prépare des petits « kolaks» (pains rituels de forme ronde) que l'on offre aux visiteurs qui viennent saluer le mort.

\section{L'enterrement (le $3^{\mathrm{e}}$ jour )}

Des mesures sont prises particulièrement :

- au moment de la mise en bière

- lors de la sortie du mort

- lors du trajet entre la maison et le cimetière : ce voyage symbolise l'itinéraire que parcourt l'âme jusque dans l'au-delà . Sur ce « chemin du mort », plusieurs arrêts ont lieu, de préférence à des croisements de routes ou au passage de ponts ; des offrandes y sont faites.

Ces arrêts figurent les « douanes » que le mort aura à passer pour arriver dans l'au-delà ${ }^{14}$.

Fig. 4 : «Assiette » vil. Prevala rég. de Montana, Bulgarie. Pain de la période de deuil (d'après laneva)

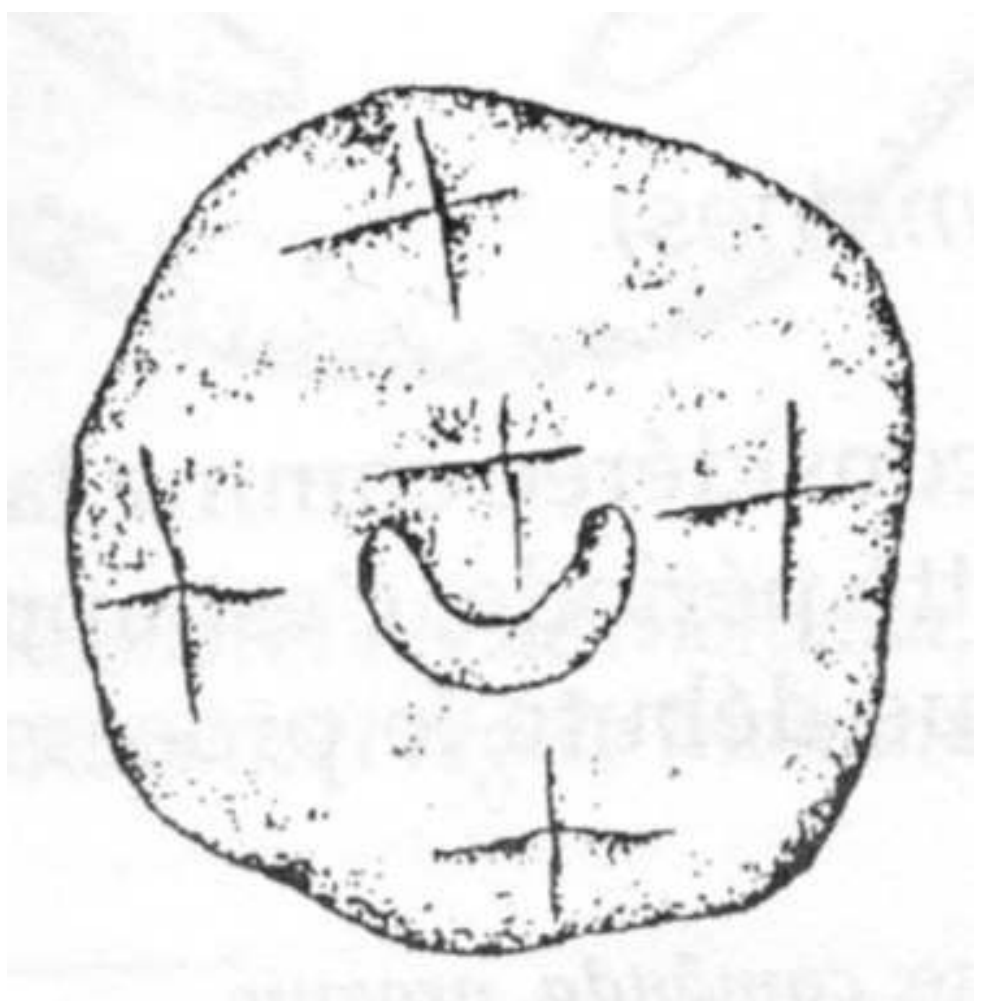

\section{Préparations céréalières}

Dans le cortège funèbre, des aides portent des paniers chargés des objets destinés à la distribution en « pomana » : y figurent pour l'essentiel : des cierges, des pains (koragu / colaci) et des « konura»;

Durant les arrêts (au moins trois), le prêtre bénit et encense le cercueil ;

En Moldavie, ces arrêts portent le nom de « ponts »; ces ponts ou « douanes » se réfèrent à la croyance selon laquelle le mort doit effectuer un voyage et passer ces «ponts » 
gardés par des « douaniers » (parfois figurés par des diables ou anges déchus : cf. Marian) qui réclament un paiement pour laisser le voyageur poursuivre son chemin vers l'au-delà. Ces « ponts » sont figurés par des pièces de tissus étendues sur le sol ; à chaque arrêt qui s'effectue ici entre le seuil de l'église et la tombe, on donne en «pomanà » les objets qui ont été placés sur le tissu : un verre de vin sur lequel est mis un petit colac dans lequel est fiché un cierge avec une pièce de monnaie. Ainsi sont "payées" les douanes pour le voyage du mort.

A l'église : c'est à l'église, après le service religieux, qu'a lieu le premier don important de nourriture : sur une table installée à cet effet, on a disposé les objets de cette offrande : des bouteilles de vin, des petits pains, une kollyva avec un cierge allumé ;

Au cimetière: sur la tombe qui sert en quelque sorte de table, on mange et boit avec le mort, en sa compagnie, on arrose sa tombe et on laisse des victuailles. On dit (bulg.) que le mort sort souvent pour boire du vin de la bouteille qu'on lui laisse ;

Sur la tombe, on casse une poterie : ce peut être la cruche qui a contenu l'eau versée sur la tombe ; une " pomanà » est distribuée par la maîtresse de maison aux fossoyeurs : elle conprend : un cierge allumé, une pièce de tissu (mouchoir) et un morceau de pain.

29 A la maison : au retour du cimetière, a lieu un repas ${ }^{15}$ auquel tous les invités participent. On y sert des mets de «jeûne » ou de "gras » selon la période de l'année où a lieu la cérémonie. Chacun reçoit un morceau de pain et un cierge à emporter chez soi.

La «table du mort: dans le sud de la Roumanie, on dresse parfois à la tête de la table principale, une petite table ronde avec une ou deux chaises destinées aux exhumés ». La petite table est donnée en pomana avant que le repas ne commence ${ }^{16}$.

\section{La quarantaine}

\section{Du $3^{\mathrm{e}}$ jour (enterrement) au $40^{\mathrm{e}}$ jour (6 semaines)}

31 La célébration du $40^{\mathrm{e}}$ jour après la mort est considérée comme la plus importante ${ }^{17}$. En effet, selon les croyances associées à cette période, c'est après six semaines que l'âme est censée s'arracher au corps et que débute le processus de putréfaction; l'âme revient une dernière fois près du cadavre, puis s'en va définitivement ${ }^{18}$. Pour cette occasion, outre les prosphora $^{19}$ (pains marqués du sceau christique) et la kollyva, principales offrandes alimentaires du cycle de deuil, on fait des pains rituels et toutes sortes de gâteaux en abondance, à distribuer avec d'autres pomana afin que le mort ait tout ce dont il a besoin pendant son voyage vers l'au-delà : libations et aliments pour assouvir soif et faim ; cierges pour éclairer son chemin et textiles (serviettes et vêtements) qui lui permettront de "payer les douanes ». En Grèce, ce moment de « décolage » de l'âme est marqué par l'offrande d'une kollyva "achevée», qui contraste avec celle «inachevée » qui l'ont précédée, où il n'y a ni sucre ni condiment ou décoration ${ }^{20}$.

32 Avant les offrandes de la "Quarantaine", ainsi que de la quarantaine à la date anniversaire de la mort, ont également lieu des pomane, notamment à 3, 6 et 9 jours ainsi qu'à 3 et 6 mois.

\section{La fin du voyage}

$\mathrm{Au}$ bout d'un an, est censée se produire l'intégration définitive du mort au pays des ancêtres; c'est aussi, pour les vivants, la fin d'une période de deuil marquée par la 
réintégration de la famille dans la communauté. En Roumanie, ce moment est symbolisé par une "danse en offrande" (hora de Pomana). C'est pour cet anniversaire que l'on prépare le plus grand plateau de kollyva très richement décoré, appelé parastas. En Bulgarie, l'achèvement du cycle est également marqué par l'offrande de pain décoré d'un rond de pâte fermé. En effet, tout au long des pomane qui ont ponctué la progression de l'âme dans son long voyage de la tombe vers l'au-delà, l'ouverture de ce cercle (appelé " petit saint Michel ») s'est rétrécit; et à l'issue de l'année de deuil, le cercle ouvert s'est transformé en un rond qu'on appelle "pleine lune", indiquant ainsi l'arrivée de l'âme dans l'au-delà.

Fig. 5 : a. « Assiette " vil. Kopilovci rég. de Montana, Bulgarie. Pain de la période de deuil (d'après Yanova 1998b). b. « Assiette » vil. Kopilovci rég. de Montana, Bulgarie. Pain du « bout de l'an » (d'après Yanova 1998b)
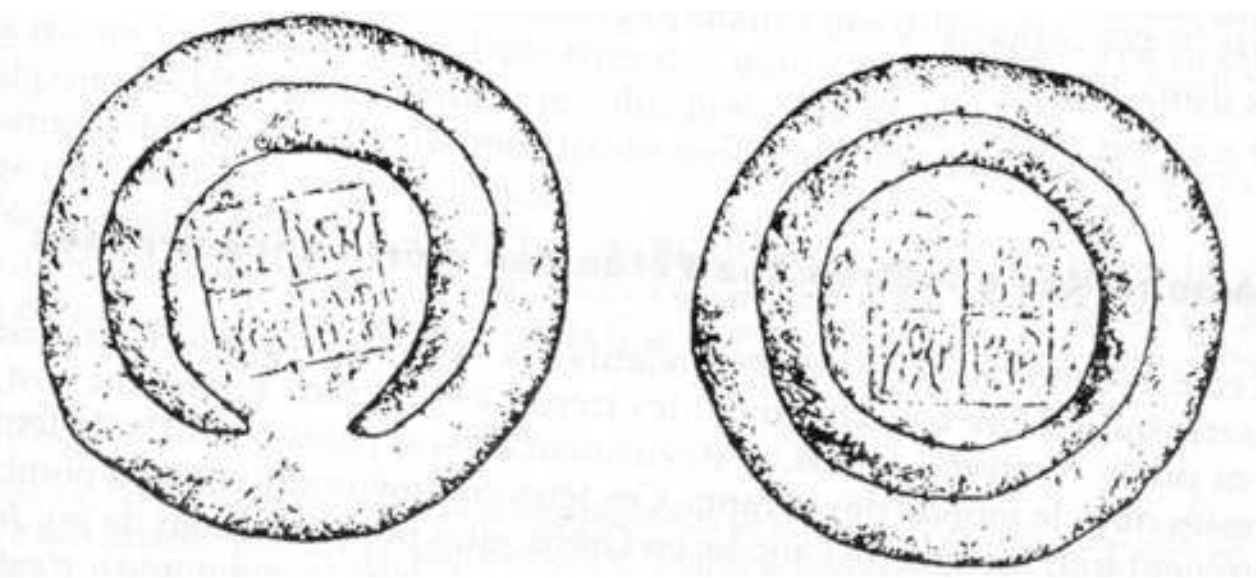

\section{Après 7 ans} réenterrés (rite auquel est associé le prêtre). Le mort n'est alors plus évoquée que comme membre du lignage à titre d'ancêtre. Ce rituel de « déterrement et de réenterrement » se fait de préférence lorsqu'un proche vient à mourir: on procède alors à la double cérémonie. Une petite table garnie de mets est donnée en pomanà pour le « déterré » (cf. plus haut, le repas du mort). progressivement achevée, qui vient symboliser la progression et l'achèvement du cycle de deuil: nous l'avons trouvée en Grèce, où le passage de l'offrande de kollyva inachevée à une kollyva complète, marque le temps fort du «décollage " de l'âme vers l'au -delà, la clôture de la première "Quarantaine ». Et nous la voyons à l'oeuvre en Bulgarie où la décoration des petits pains progresse du cercle ouvert au cercle fermé, en marquant la clôture un an après le décès, pour le parastas anniversaire.

Ainsi donc, la kollyva est « achevée » et la boucle du kolak bouclée. Les vivants ont honoré les morts, et les morts sont devenus des ancêtres.

Mais revenons précisément à cette image de l'offrande à la forme imparfaite et Ces deux exemples se situent ainsi aux deux stations importantes du périple de l'âme: son départ du monde d'ici et son arrivée dans l'au-delà. 
Fig. 6 : « Grand-père et grand-mère », vil. Mladovo rég. de Sliven, Bulgarie. Pain de commémoration (d'après Yaneva 1989a)

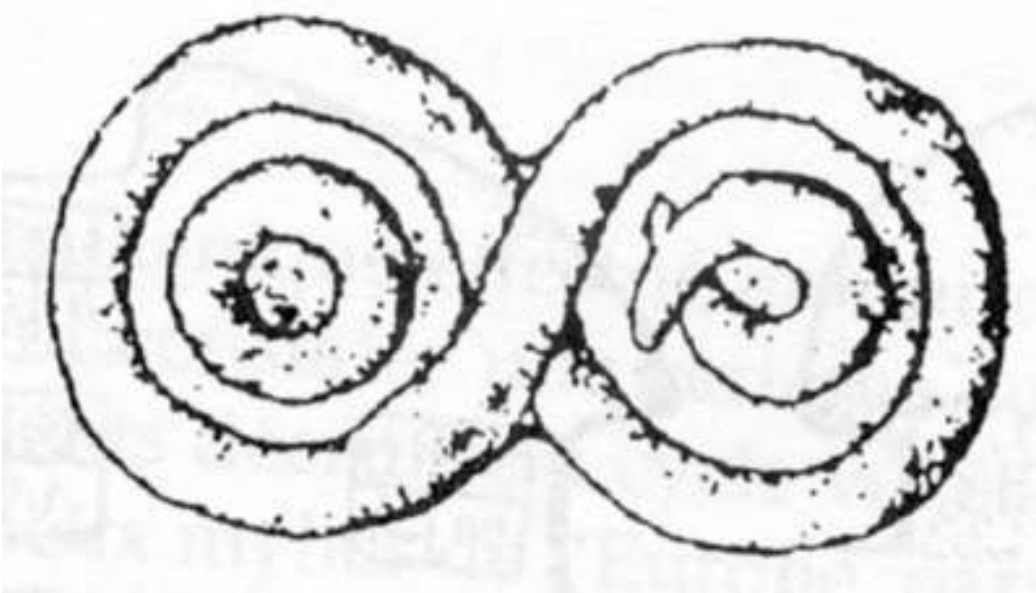

On retrouve une variante de cette même représentation du cheminement vers l'au-delà qui s'oppose à l'arrêt définitif, dans le fait que les offrandes de kolaks doivent toujours être en nombre impair. D'une manière plus générale, en dehors des rites de deuils, cette opposition entre pair et impair symbolise la mouvance, le déplacement, la vie, dans son opposition à l'équilibre, l'absence de mouvement, la mort. C'est ce qui explique pourquoi, dans les sociétés balkaniques, il faut être attentif, lorsqu'on fait un cadeau (et pas seulement une offrande), à respecter la règle du nombre impair.

\section{La mémoire des ancêtres : les Fêtes des morts ancestralisés}

\begin{abstract}
à travers, au-delà, et deviennent ainsi séparés à jamais de leurs proches restés dans le monde des vivants. Ces fêtes commémoratives sont nombreuses et s'égrènent tout au long de l'année ; en Grèce, elles portent le nom de psychosabbata, «samedi des âmes ", en Bulgarie, celui de zadusnici, c'est-à-dire «samedis morts », et en Roumanie celui de mosi, ayant ici le sens d'ancêtres.

A côté de cette catégorie de rites relatifs à la mort individuelle, il en existe une autre série qui célèbre collectivement les trépassés, au sens littéral du mot, ceux qui sont passés
\end{abstract}

\section{Dans ce pays, on assiste à une véritable prolifération de telles fêtes d'ancêtres qui en viennent à couvrir pratiquement toute l'année. Mais ces commémorations occupent aussi une place importante dans l'ensemble des cultures balkaniques. On peut aisément y compter une dizaine de Samedi des âmes ${ }^{23}$. Comme pour les rites de deuils, ces commémorations sont l'occasion de faire des offrandes destinées à l'au-delà, et plus particulièrement aux ancêtres du lignage. Partout, on y rencontre des offrandes céréalières sous forme de kollyva et de petits pains de blé y occupent une place centrale. On y ajoute des lukoums, divers gâteaux et sucreries, du vin et des fleurs. Si l'on peut}


observer d'un lieu à l'autre de nombreuses variantes dans la composition de telles offrandes, certaines de ces fêtes ont cependant en commun de requérir la fabrication de mêmes pains spécifiques ${ }^{24}$.

Le calendrier liturgique officiel commun aux pays de l'orthodoxie balkanique reconnait trois grandes fêtes des Morts. Ce sont :

- le samedi qui précède la Saint -Archange (Michel, le 8 novembre), dont la première date possible de célébration coïncide avec la fête des morts catholique ( 2 novembre, lendemain de la Toussaint).

- le samedi avant la «Semaine blanche » qui inaugure le carême orthodoxe stricte ${ }^{25}$.

- le samedi qui précède la Pentecôte.

On notera cependant que les traditions populaires des différents pays n'accordent pas la même importance à ces trois fêtes des morts préconisées par l'Eglise. Ainsi en Bulgarie, les fêtes généralement les plus honorées sont:

- le « samedi des morts » d'automne, dédiée aux « Oubliés » : l'offrande est ici un pain allongé dit « pain de Saint -Archange $»^{26}$.

- la fête des âmes du temps des cerises, ainsi appelé vu qu'il coïncide plus ou moins avec la maturation de ce fruit. Cette commémoration participe du cycle post-pascal et correspond à la fête de l'Ascension (40 jours après Pâques), mais peut être repoussée jusqu'à la Pentecôte (donc 10 jours plus tard). Dans les deux cas, la commémoration coïncide avec la période de séjour des ancêtres sur terre ${ }^{27}$.

Fig. 7 : a. "Assiette de St-Michel », vil. Cerven, Bulgarie. Pain de commémoration (d'après Marinov). b. «Assiette de St-Michel », vil. Kneza, Bulgarie. Pain d'enterrement et de commémoration (d'après Marinov)

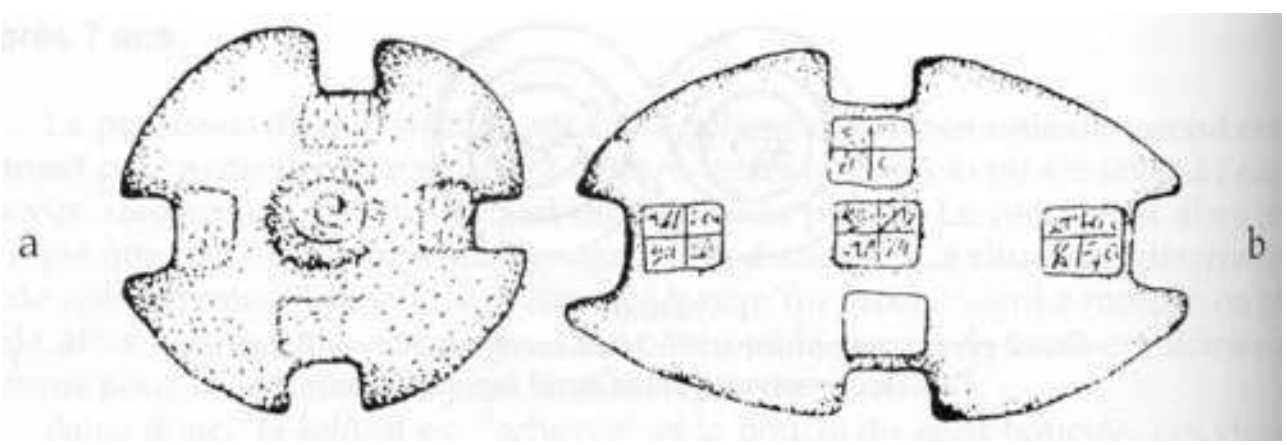

Pour les Roumains, l'accent est mis précisément sur ces moments d'arrivée et de départ des ancêtres : les deux rites commémoratifs les plus importants se situent dès lors aux "Ancêtres de Printemps» (Mosi de Primavara), dont la fête ouvre le grand carême de Pâques, et aux « Ancêtres de la Pentecôte » (Mosi de Rusalii). L'abondance et la richesse de telles offrandes mortuaires rendent impossible une description même sommaire des fêtes des mosi roumains dont le chiffre peut s'élever jusqu'à une vingtaine par année .

Contentons-nous de souligner les traits communs des offrandes céréalières qui accompagnent ces célébrations. Comme en Bulgarie et en Grèce, elles consistent pour l'essentiel en petits pains et de kollyva (blé bouilli et sucré). Le prototype de la pomana roumaine se présente comme la combinaison de trois éléments qui correspondent en quelque sorte, aux trois « offrandes fondamentales » à même de répondre aux besoins des ancêtres: l'eau (et/ou le vin), le pain et la lumière. Chaque famille prépare autant d'offrandes qu'il y a d'ancêtres à honorer dans le lignage et chacune se compose d'une petite cruche en terre cuite qui doit être neuve ${ }^{28}$, et qui contient l'eau ou le vin ; sur cette 
cruche dont on garnit l'anse de quelques fleurs, est posé un petit pain dans lequel est fiché un cierge. Cette triple pomana, ou plus particulièrement le petit pain qui y figure, porte parfois le nom de cap, littéralement « tête ». C'est, en quelque sorte, chaque ancêtre qui est ainsi figuré par une cruche et un petit pain orné de son cierge.

Enfin, il faut encore mentionner dans ces traditions roumaines de commémorations, l'usage qui consiste à confectionner un pain destiné aux personnes " connues et inconnues, oubliées ou non oubliés, visibles et invisibles $»^{29}$. C'est l'offrande « oubliée » (uitata). Sa forme varie selon les régions et les dates de son emploi ; aux Quarante Martyrs du 9 mars, par exemple, ce pain reçoit souvent la forme d'un visage humain, avec des oreilles, un nez, mais pas d'yeux.

\section{Si le grain ne meurt...}

49 A l'issue de ce périple balkanique effectué autour de l'année commémorative des âmes ancestrales, on peut constater une certaine concordance entre le calendrier de ces fêtes et les moments décisifs du cycle végétatif des céréales. C'est déjà ce que nous disent les plus vieux mythes de l'Europe, par exemple, lorsque Déméter pleurant sa fille perdue, arrête le cycle végétatif et affame les hommes privés de blé. C'est encore ce que soulignent les rites grecs lorsque se déroulent les célébrations des panspermia, associées au développement saisonnier de céréales et légumineuses.

Ainsi, l'activité rituelle liée à la commémoration des ancêtres a tendance à s'endormir pendant que meurt la nature végétale. Et elle renaît avec les signes annonciateurs du printemps, les hirondelles, par exemple, ou les crocus et les huppes des "Quarante Martyrs ", qui, en Roumanie, prêtent parmi d'autres, leur nom aux petits pains confectionnés à cette date. Les rites se bousculent durant toute la période de germination, de verdoiement et de mûrissement des fruits de la terre ${ }^{30}$, pour se raréfier avec la saison d'hiver, lorsque le blé d'automne a été mis en terre.

\section{BIBLIOGRAPHIE}

ANDREESCO, I. \& BACOU, M., 1986, Mourir à l'ombre des Carpates, Paris, Payot.

BAILLY, M.A., 1928, Dictionnaire Grec-Français. Paris, Hachette

BERNABE, J., 1980, Le symbolisme de la mort. Croyances et rites roumains. Gent, Communication and cognition.

LE GOFF, J., 1981, La naissance du Purgatoire. Paris, Gallimard.

MARIAN, S. Fl., 1892. L'enterrement chez les Roumains, étude ethnographique. Bucuresti, ed. Academiei Române.

MESNIL, M., 1983, « Vampirisme et tradiction orale en Roumanie : les sources populaires d'un mythe savant ». Dialogue, Montpellier, $\mathrm{n}^{\circ} 10-11:$ 87-98. 
MESNIL, M., août 1985, « Chemin des morts, chemin des âmes. Deux représentations d'une même

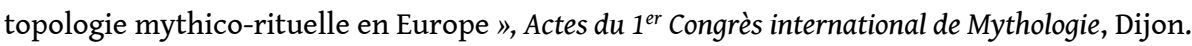

MESNIL, M., 1990, « Revenants et sorciers : entre vie et mort, croyances, rites et récits de Roumanie ». In : Chanter la mort. (I. Andreesco ed.) Cahiers de Littérature Orale, nº 27 : 175-94.

MESNIL, M. et POPOVA, A., 1990, «Des ancêtres aux nouveaux nés. Les pains de la Saint -Quarante » (en collab. avec A. PopovA), L'Uomo, Rome, IIIn.s., n¹.

MUSLEA, I. \& BIRLEA, O., 1970, Typologie du folklore d'après les réponses au questionnaire de B.P. Hasdeu, Bucuresti, Editura Minerva.

NICULITA-VORONCA, E., 1903, Coutumes et croyances du peuple roumain réunies et établies dans un ordre mythologique., Cernàuti, Tip. Isidor Wiegler.

POP, M., « Le mythe du « grand voyage » dans les chants des cérémonies funèbres roumaines ». I $\mathrm{n}$ : To honor Roman Jakobson. Essays on the occasion of his seventieth birthday-11oct. 1966, vol. II, Paris-The Hague, Mouton 1967 : 1602-1609.

POPOVA, A., « Ni chair, ni poisson : Tryphon le Coupé ». In : Cahiers de Littérature Orale, vol. 3, 1977.

STAHL, P.-H., - serie nouà 1983-, L'autre monde. Les signes de reconnaissance. In : Buletinul bibliotecii române . Freiburg (vol X (XIV) 108).

VAN GENNEP, A., 1909, Les rites de passage. Paris, E. Nourry.

VULPESCO, M., 1928, Les coutumes roumaines périodiques. Paris, ed. Larose.

\section{NOTES}

1. Le Goff, op. cit. : 69 .

2. L'expression (stari mârtvi) c'est-à-dire « vieux morts, morts anciens » est une désignation de la fête des morts moins répandue que le terme de zadusnica, mais significative pour notre propos.

3. Pour des exemples de tels rituels observés au cours des dix dernières années, voir notamment : Andreescou et Bakou, op. cit. : 108.

4. Archives de l'Université de Sofia, Enquêtes de terrain, Boneva, vil. Xvojna, Rhodope Central ; sans date, mais sans doute pas avant les années 80 .

5. Voir à ce propos Mesnil et Popova, 1990. La kollyva se prépare de la manière suivante : on prend la quantité voulue de froment, compte tenu que les grains vont tripler de volume au cours de la cuisson. On asperge les grains d'un peu d'eau afin de les ramollir. Puis on les pile dans un grand mortier en bois (piua en roumain), afin de séparer le grain de la balle. Ensuite on lave les grains deux ou trois fois et on les fait bouillir en y ajoutant un peu de sel. Une fois bouillie, la préparation est versée sur un grand plateau pour la laisser quelque peu sécher. On y ajoute des noix, des fruits secs, un zeste de citron et du sucre. Ainsi prête, la kollyva est versée dans un grand plat et saupoudrée de sucre ; on y dessine un cercle dans lequel on inscrit une croix ; on y plante un cierge. La kollyva peut alors être portée à l'église. (De nos jours, dans les villes, on peut acheter directement la kollyva à l'église). Cette préparation doit être consommée rapidement, car elle fermente très vite.

6. In : 12,24 .

7. Voir Dictionnaire Bailly. Une telle évolution de dons de friandises en monnaie, est perceptible de nos jours dans les traditions balkaniques observées lors des quêtes d'enfants, notamment au Nouvel An.

8. voir notre article dans ce volume. 
9. Losanova, G., "Le pain des morts", in Le pain dans les traditions slaves., Sofia, Institut d'Ethnographie et Musée, 1997 : 37-48

10. Ainsi par exemple, on ne fabriquera jamais un levain à partir de la pâte qui a servi à la confection des pains rituels destinés à un mort. Marian, S.F., op. cit. : 372

11. Le «sceau christique " existe dans une version « domestique » (simplifiée par rapport au sceau «eucharistique » utilisé par le prêtre), il s'agit d'un carré (correspondant au "saint agneau ») dans lequel s'inscrit une croix; dans chaque carré ainsi limité par la croix, sont inscrites les lettres suivantes IS XR /Ni KA, qui signifient en grec « Jésus Christ a vaincu » (voir aussi la Fig. 1).

Chaque famille possède le sien et c'est la maîtresse de maison qui l'utilise ; les noms donnés à cet objet, pistornic en Valachie; prescornic en Moldavie; prosfornik/preskurnik en bulg., ont pour étymologie commune le mot *prosphora, offrande, nom donné au pain eucharistique, prototype du «pain marqué ".

12. voir Mesnil et Popova, 1990

13. Stanka Yaneva, 1989a

14. voir Mesnil, 1985

15. En Roumanie, on l'appelle souvent lui-même pomana, ou encore : comânda, praznic.

16. Bernabé : 85

17. En bulgare, l'offrande de la quarantaine est toujours indiquée par les dénominations : "quarantaine ", la «grande libation de la tombe ( traduction approximative de prelivane na groba), et « nivellement de la tombe » (ravnenie).

18. Mais si l'on n'a pas fait les offrandes nécessaires, l'âme revient tourmenter les vivants et réclamer son dû (Mesnil 1983).

19. En Bulgarie, le nombre des prosphora données en offrande correspond généralement au nombre des jours écoulés depuis la mort.

20. A. Popova, enquête de terrain en Grèce du Nord, 1996.

21. En Roumanie, il existe une "prière de déliement » que prononce le prêtre si le corps n'est pas complètement putréfié. La période de 7 ans constitue la norme; en pratique, elle diffère, notamment en fonction des décès qui surviennent parmi les proches parents, et la cérémonie peut avoir lieu après 3 ou 5 ans, voire 9 ans selon le cas.

22. Ce sont les paroles que le réalisateur fait dire au prêtre d'Ohrid, au début et à la fin de son film « Before the rain » (1994).

23. Petrov., op. cit.

24. En Bulgarie, ces pains des ancêtres sont habituellement de forme allongée, décorés d'une croix sculptée en pâte; il sont appelés "pains allongé, pains des oubliés, pain de la mort. En Roumanie, leur nom varie selon la date du cycle pour laquelle ils sont confectionnés. Figurent dans cette liste, le nom de "mosi ", ancêtres (donné à l'ensemble de ces commémorations), mais aussi des noms d'oiseaux (huppe) ou de plantes (petits crocus) etc.

25. La semaine blanche est vouée au végétarisme "modéré »: les viandes sont prohibées, mais la consommation des laitages, du beurre et des fromages est plutôt recommandée. Le carême strict inauguré par la Semaine de Théodore interdit la consommation de tout produit d'origine animale.

26. Samedi qui précède la Saint -Michel ( 8 novembre) ou parfois, la Saint-Dimitri (26 octobre). La forme oblogue des pains est rare en Bulgarie comme en Roumanie (cf. T. Agapkina, 1997 : 48-62). Elle indique les destinataires de cette sorte d'offrandes : «Oubliés » du Samedi des âmes de la Saint -Michel ; « Oubliés » des « Mosi de printemps » (9 mars) (voir ci-après).

27. Cette période qui commence avant Pâques («Grand Jeudi» ou dimanche des Rameaux) se clôture au plus tard à la Pentecôte. Cette offrande post -pascale est une manière de prendre congé des ancêtres. 
28. C'est ce qui explique l'existence de foires aux céramiques de printemps, appelées « Mosi » : les familles s'y approvisionnent de cruches neuves en vue de telles offrandes.

29. T. Agapkina, op. cit. : 48-62

30. C'est à l'approche de Pâques et jusqu'à la Pentecôte que les ancêtres sont censés revenir visiter les lieux où ils ont vécu.

\section{RÉSUMÉS}

L'article tente de procéder à une mise en contexte des préparations céréalières contemporaines destinées aux offrandes mortuaires dans l'aire culturelle de l'orthodoxie balkanique. En effet, les rites dans lesquels prennent place ces offrandes font référence à une conception de la mort qui diffère de notre conception occidentale moderne: la mort est pensée ici comme un long processus dont l'aboutissement est marqué par la transformation du mort en ancêtre. Dès lors, on distinguera deux types de cycles rituels. Le premier concerne la mort individuelle. Cette période s'étale sur un an, de l'enterrement à la cérémonie de clôture du deuil. L'autre cycle correspond aux commémorations des ancêtres et se déroule au rythme des saisons. Les préparations liées à ces cérémonies comprennent la bouillie de blé (kollyva) et différentes sortes de pains dont la forme, l'ornementation et/ou l'appellation indiquent leur destination spécifique.

The article makes an attempt to understand the context of contemporaneous cereal preparations for the dead in the orthodox cultural area of the Balkans. The rituals in which these offerings occur refer to a perception of death that differs from the western modern perception: death is thought of as a long process that ends with the transformation of the dead into an ancestor. Two types of ritual will thus be distinguished. The first one concerns individual death. This period covers one year, from the burial until the ceremony of grief. The other cycle is concerned with the commemoration of the ancestors and takes place according to a seasonal rhythm. The preparations related to these ceremonies include a porridge made of corn (kollyva) and different sorts of bread whose form, ornamentation and/or name are indicative of

\section{INDEX}

Mots-clés : Pain, céréales, rites funéraires, Balkans

Keywords : Bread, cereals, funerary rites, Balkans

\section{AUTEURS}

\section{MARIANNE MESNIL}

Centre de Recherche en Ethnologie Européenne, Institut de Sociologie, Université Libre de Bruxelles, Belgique. 
ASSIA POPOVA

LACITO, CNRS-Paris, France. 